\title{
Qualifikationen für die digitale Ökonomie
}

\author{
Robert Winter, Ulrike Baumöl \\ Institut für Wirtschaftsinformatik und \\ Executive MBA in Business Engineering, Universität St. Gallen \\ Müller-Friedberg-Strasse 8, CH-9000 St. Gallen \\ Robert.Winter@unisg.ch, Ulrike.Baumoel@unisg.ch
}

\section{Herausforderungen der digitalen Ökonomie}

Die vielbeschworene digitale Ökonomie hat - trotz einiger Rückschläge zu einer immer schnelleren und oftmals radikalen Veränderung der Strukturen von Unternehmen in vielen Branchen beigetragen. Diese Veränderungen haben nicht nur die ohnehin schon ,volatile“ Bereiche getroffen, sondern auch diejenigen Kernelemente erfasst, die lange als stabil betrachtet wurden. Während früher häufig bereits eine Produktinnovation für die erforderliche Anpassung ausreichend war, müssen heute zum Teil tiefgreifende Prozessinnovationen umgesetzt werden, damit dem Veränderungsbedarf Rechnung getragen werden kann. Für die betroffenen Unternehmen bedingt das einen grossen und vor allem komplexen Anpassungsbedarf. So hat sich beispielsweise der (Retail-) Finanzdienstleistungsbereich in den letzten Jahren grundlegend gewandelt. Das seit einem Jahrhundert oder mehr bewährte und an der „Bankproduktion“ ausgerichtete Universalbankkonzept wird nun durch einige wenige grosse „Bankfabriken“, sogenannte Transaktionsbanken, konkurrenziert, die in einem offenen flexiblen Wertschöpfungsnetzwerk mit vielen „Spezialisten“ (z.B. für die Produktentwicklung) und vor allem mit kundenorientierten Vertriebspartnern zusammenarbeiten.

Auslöser, Treiber und „Enabler“ dieses Wandels, der gleichermassen Strategie, Prozesse, Führung, Unternehmenskultur und Informationssysteme betrifft (Kelly, 1998), können meistens im Bereich der Informations- und Kommunikationstechnologie lokalisiert werden (Evans, Wurster, 1999).

Obwohl Deregulierung und Globalisierung ebenfalls eine wichtige Rolle in der Transformation $\mathrm{zu}$ vernetzten Wertschöpfungsnetzwerken spielen, wird beispielsweise die Veränderung im Finanzdienstleistungsbereich we- 
sentlich durch neue und innovative Technologien ermöglicht: „Persönliche“ Zugangsgeräte (PC, PDA, Mobiltelefon) erlauben einen durchgehenden, u.U. mobilen Zugriff auf Leistungen, neue Standard-Anwendungssoftware im Finanzdienstleistungsbereich unterstützt die effiziente Leistungserstellung, die Automatisierung von Märkten mit Hilfe geeigneter IT-Systeme und mächtige Datenintegrations- und -auswertungswerkzeuge bringen Kunde und Dienstleister näher zusammen. Neue Zugangskanäle, zunehmende Virtualisierung und vor allem die Herausbildung von Standards für das Business Networking (Österle, Fleisch, Alt, 2001) erlauben eine weitgehende Trennung zwischen der Produktion und dem Vertrieb von Finanzdienstleistungen.

Aus diesen Veränderungen entstehen erhebliche Herausforderungen für Unternehmen und als Konsequenz auch für die hauptsächlich betroffene Disziplin Betriebswirtschaftslehre. Völlig neue Geschäftsmodelle entstehen, während traditionelle Geschäftsmodelle verschwinden. Integration und Automatisierung erfordern nicht nur den radikalen Neuentwurf der (internen) Geschäftsprozesse (Davenport, 1993). Die Unternehmensführung muss sich vielmehr auch mit der Gestaltung der Beziehungen mit Netzwerkpartnern, der Gestaltung unternehmensübergreifender Prozesse und der ,änderungsfreundlichen“ Gestaltung der Informationssysteme auseinandersetzen.

Darüber hinaus ist zusätzlich zum traditionellen Branchenwissen eine intensive Verfolgung (und ein fundiertes Verständnis) der Innovationen der Informations- und Kommunikationstechnik unabdingbar, um Geschäftspotenziale bewerten zu können. Schliesslich sind „soft skills“ erforderlich, um die Konsequenzen für die Unternehmenskultur erkennen und verstehen zu können sowie die Wahrnehmung der Stakeholder für die Veränderungen zu sensibilisieren und damit eine Verhaltensänderung zu motivieren. Die Vielgestaltigkeit und Komplexität der Veränderungsprozesse hat zur Folge, dass die Gestaltung und Führung dieser Prozesse weder disziplinär (z.B. allein durch Betriebswirte oder Informatiker) noch funktionsbezogen (z.B. Strategieentwicklung vs. Marktbereiche vs. Zentralfunktionen) erfolgen kann. Dazu bedarf es statt dessen ganzheitlich denkender und umfassend qualifizierter „Architekten des Wandels“, die alle Dimensionen der Veränderungen und ihre Zusammenhänge verstehen und daraus eine stabile, aber dennoch agile neue Unternehmensstruktur entwickeln können.

Die skizzierten Herausforderungen der digitalen Ökonomie schaffen Handlungsbedarf sowohl für betriebliche Weiterbildung als auch für Einrichtungen der tertiären Bildung. Erstausbildungen fokussieren auf die Vermittlung disziplinärer Inhalte wie beispielsweise Betriebswirtschaftslehre oder Informatik. Aufgrund der höheren Anforderungen, insbesondere 
hinsichtlich eigener Erfahrungen und Sozialkompetenz, werden interdisziplinäre Inhalte zumeist erst in Nachdiplomstudiengängen oder Aufbauprogrammen vermittelt.

In diesem Beitrag werden Qualifikationsprofile für „Architekten der Veränderung“ entwickelt. Im Mittelpunkt steht das Qualifikationsprofil „Business Engineer“, für das ein Ausbildungskonzept abgeleitet wird. Parallel zum Konzept des „Enterprise Engineer“ nach Martin (1995) wurde Business Engineering Mitte der 1990er Jahre als ganzheitlicher Methodenansatz für die Konzeptualisierung, den Entwurf und die Umsetzung neuer Geschäftsmodelle entwickelt, die im wesentlichen durch IT-Innovationen ermöglicht werden (Österle, 1995; Österle, Winter, 2003). „Ganzheitlich“ heisst in diesem Zusammenhang einerseits, dass sich die methodische Unterstützung nicht auf Informationssystementwicklung beschränkt, sondern dass auch Strategie- und Organisationsentwicklung sowie Prozessmanagement unterstützt werden. Andererseits muss ein ganzheitlicher Ansatz nicht nur fachliche Aspekte von Veränderungsprozessen abdecken, sondern auch die kulturellen und politischen Dimensionen des Wandels berücksichtigen.

\section{Rollenmodelle in der Veränderung}

Veränderungen werden, wie bereits oben beschrieben, insbesondere durch Innovationen der Informations- und Kommunikationstechnik ausgelöst. Erkannt, getrieben, umgesetzt und gelebt werden Veränderungen jedoch von den Menschen in den Unternehmen. Deshalb bringt die Veränderung verschiedene Rollenmodelle hervor, die für einen erfolgreichen Transformationsprozess unabdingbar sind. Jedes dieser verschiedenen Rollenmodelle erfüllt spezifische Anforderungen, die in den einzelnen Phasen des Veränderungsprozesses zum Tragen kommen. Grundsätzlich gibt es eine Vielzahl von Möglichkeiten, den Veränderungsprozess bzw. Veränderungswellen zu strukturieren und die einzelnen Phasen zu benennen.

Die Ziele und Aufgaben der grundlegenden Rollenmodelle beziehen sich auf die verschiedenen Phasen, unabhängig davon, wie viele Phasen definiert werden. Das gesamte Rollenportfolio umfasst die folgenden Profile (Baumöl, Winter, 2003):

Treiber und Unterstützer' ${ }^{I}$ Diese Rolle ist am besten für einen Business Engineer geeignet, der Mitglied der Geschäftsleitung eines Unterneh-

1 In diesem Beitrag wird aus Gründen der Lesbarkeit auf eine Nennung der weiblichen Form der jeweiligen Bezeichnungen verzichtet. Auch wenn nur die 
mens ist. Während die Identifikation des Veränderungsbedarfs auch durch andere Rollen bzw. Mitarbeiter erfolgen kann, müssen die Initiierung und das Vorantreiben von Veränderungen aus dem TopManagement kommen. Nur so ist sichergestellt, dass neben der entsprechenden Unterstützung auch der politische Rückhalt für das Projekt gegeben ist.

Architekt: Das ist die klassische Rolle des Business Engineers: Er übernimmt die Gestaltung des Veränderungsprozesses aus einer ganzheitlichen Sicht. Das bedeutet, dass er die Ziele setzt, Vorgaben über die Zielerreichung macht, die Ressourcen plant und entsprechend einsetzt, Ergebnisse überprüft und an die Geschäftsleitung berichtet sowie den gesamten Prozess führend begleitet - sowohl aus der fachlichen Sicht als auch aus der Sicht der Organisationsrestrukturierung und des Kulturwandels. In den verschiedenen Perspektiven des daraus resultierenden Berufsbilds werden die Kompetenzen und Fähigkeiten, die diese Rolle auszeichnen, noch einmal näher erläutert. Eine wichtige Charakteristik für das Rollenbild des Architekten ist, dass gerade diese Rolle weitere Rollen integriert: Der Architekt nimmt zugleich Aufgaben eines Managers, eines Personalentwicklers, eines Controllers, eines Marketingexperten und ggf. noch eines IT-Managers wahr.

Implementierer: Der Implementierer ist vor allem durch eine hohe fachliche Kompetenz gekennzeichnet. Im Rahmen dieser Rolle muss zwar auch ein Gesamtüberblick über den gesamten Transformationsprozess gegeben sein, damit die Konsequenzen des eigenen Handelns im Gesamtzusammenhang klar sind. Primäre Aufgabe des Implementierers ist aber nicht eine aktive Gestaltung des übergeordneten Prozesses, sondern vielmehr die Erfüllung der ihm gesetzten Ziele unter Berücksichtigung der Gesamtaufgabe.

Bewahrer und Entwickler: Nach Abschluss des Projektes müssen die neuen Strukturen und auch die veränderte Kultur weiterhin gelebt bzw. auf ihre Validität überprüft und ggf. an kleinere Veränderungen angepasst werden. Die Bedeutung dieser Rolle ist nicht zu unterschätzen, weil an ihr ein wesentlicher Teil der Glaubwürdigkeit der Veränderungsauslöser hängt: Vor allem die Mitarbeiter, die nicht unmittelbar und aktiv in dem Projekt mitgearbeitet haben, beobachten ihr neues Umfeld und erwarten ein „Vorleben“, wenn nicht sogar eine Überprüfung im Sinne einer Bewahrung und Umsetzung der neu gesetzten Massstäbe und Ideen. Genau wie die Rolle des Architekten ist diese Rolle temporär

männliche Form verwendet wird, ist die weibliche Form immer mit eingeschlossen. 
begrenzt; Sie wird nur solange aktiv „gelebt“, bis wieder eine neue Transformationswelle einsetzt.

Für die Veränderungen der Wirtschaft, die die digitale Ökonomie mit sich bringt, kommt den Business Engineers als Spezialisten für die Veränderung besondere Bedeutung zu. Während andere Gruppen vorbereitende, begleitende, unterstützende und/oder ausführende Rollen im Veränderungsprozess wahrnehmen, sollen Business Engineers Veränderungsprozesse massgeblich und ganzheitlich gestalten bzw. führen. Die Hauptlast dieser Aufgaben wird je nach Organisationsform des betreffenden Unternehmens und je nach Branche bzw. Situation des Unternehmens durch eine Kombination interner Berater bzw. Projektleiter und externer Berater getragen. Daneben finden sich (z.B. in Unternehmensbereichen mit hohem Veränderungsgrad) Business Engineers auch in Linien-Führungspositionen oder in sich stark verändernden Branchen sogar als Unternehmensgründer.

\section{Der Business Engineer}

Das Qualifikationsprofil des Business Engineers wird im wesentlichen bestimmt durch vier Aspekte, die in Einklang zu bringen sind, damit der Transformationsprozess erfolgreich führbar wird. Diese vier Aspekte umfassen in der fachlichen Dimension des Business Engineering-Modells [siehe z.B. Österle, Winter 2003] zum einen die strategische Zielerreichung, die durch das Setzen von mittel- bis langfristigen Zielen und die Vorgabe der Meilensteine auf dem Weg dahin (z.B. klar formulierte Zielvereinbarungen und die Vorgabe von Qualitätsstandards) gekennzeichnet ist. Zum anderen muss der Business Engineer die Ebene der Arbeitsabläufe und der Organisation so gestalten, dass im Hinblick auf die Zielerreichung optimale Bedingungen (z.B. im Hinblick auf eine Ressourcenallokation, aber auch auf eine leistungsgerechte Entlohnung) geschaffen werden. Die politisch-kulturelle Dimension wird einerseits durch die Betrachtung der Führungskompetenzen und andererseits durch die explizite Berücksichtigung der kommunikativen Kompetenzen des Business Engineers einbezogen. Im Rahmen der Führungskompetenzen werden sowohl die eigene Führung (im Sinne eines Selbstmanagements) als auch die Führung der Personen behandelt, die an dem Veränderungsprozess beteiligt und damit letztlich auch von ihm betroffen sind.

Nachfolgend werden die Kernpunkte dieser vier Aspekte kurz erläutert (Baumöl, Winter, 2003):

Strategische Entscheidungen im Hinblick auf die zu erreichenden Ziele im Veränderungsprojekt und die Ableitung des Vorgehens gehören zu 
den grundlegenden Aufgaben des Business Engineers. Darüber hinaus muss im Sinne eines „Blicks über den Tellerrand“ auch die Zeit nach dem Veränderungsprojekt in Betracht gezogen und in die Strategiebildung integriert werden. Demzufolge ist in diesem Bereich sowohl die fachliche als auch die strategische Kompetenz des Business Engineers gefordert. Weitere Zielsetzungen, die zu definieren und zu berücksichtigen sind, beziehen sich zum einen auf das Mass an zu erreichender Qualität der Projektergebnisse und die frühzeitige Sicherstellung der Realisierung. Zum anderen muss festgelegt werden, welche internen und / oder externen Anspruchsgruppen adressiert werden und wie diese hinreichend in der Strategiebildung berücksichtigt werden können.

Die fachliche Perspektive umfasst z.B. die Arbeitsprozesse und die Organisation des Transformationsprozesses. Der Business Engineer koordiniert den Ressourceneinsatz sowie die Verteilung der Aufgaben und entscheidet, welche Technologien wann zum Einsatz kommen sollen. Die optimale Erfüllung dieser Aufgaben erfordert eine hohe fachliche Kompetenz, die durch eine entsprechende methodische Kompetenz ergänzt wird.

Die Führungsrolle des Business Engineers fordert nicht nur soziale, sondern auch strategische Kompetenzen. Das bedeutet, dass die Führung im Rahmen des Veränderungsprozesses aus der Sicht der sozialen Kompetenz das aktuelle Arbeitsumfeld so gestalten muss, dass eine maximale Motivation und damit auch Zufriedenheit möglich wird. Die strategische Kompetenz ergänzt dieses Bild mit einer zukunftsgerichteten Entwicklung der Fähigkeiten der Mitarbeiter und gibt ihnen damit eine Vision im Hinblick auf ihre zukünftigen Aufgaben und Ziele im Unternehmen. Ein integraler Bestandteil der Führungsrolle ist aber auch, wie bereits erwähnt, die „Selbstführung“ des Business Engineers. Nur eine permanente Reflexion des eigenen Verhaltens und hohe Disziplin in der Verfolgung der gesetzten Ziele schafft Vertrauen in die Person und erzeugt die Glaubwürdigkeit, die für die Führung durch einen erfolgreichen Veränderungsprozess unabdingbar ist. Erfolg in einem Veränderungsprozess bedeutet also nicht nur ,wirtschaftlichen“ Erfolg, also z.B. die Realisierung von Einsparungspotenzialen im Rahmen eines Mergers, sondern auch die Akzeptanz der veränderten Situation durch die Mitarbeiter und dadurch auch das „Mittragen“ des Prozesses und seines Ergebnisses.

Eng mit dem vorherigen Punkt verbunden sind auch die Fähigkeiten des Business Engineers zur Kommunikation sowie zur Gestaltung einer spezifischen Veränderungskultur. Die methodisch geführte Interaktion prägt gleichzeitig das soziale Arbeitsumfeld, z.B. durch Teamarbeit zum richtigen Zeitpunkt und nicht um jeden Preis, das richtige Mass an In- 
formation und Kommunikation und eine gut dosierte Konfliktbearbeitung, die weder Konflikte ignoriert noch sie permanent thematisiert. Dabei muss das Kommunikationsverhalten authentisch sein, damit eine vertrauensvolle Interaktion entsteht.

Die Basisqualifikation des Business Engineers sind betriebswirtschaftliche Fachkenntnisse, d.h. sowohl generelle Betriebswirtschafts- und Managementkompetenzen als auch Branchenkenntnisse. Um neuartige Geschäftslösungen konzipieren zu können, benötigt er darüber hinaus eine Vision, wie Unternehmen im Informationszeitalter aussehen sollten und welche Regeln für das Geschäft in Zukunft gelten werden. Dazu muss er die Informations- und Kommunikationstechnologie so weit verstehen, dass er sie zur Konzeption neuer Geschäftslösungen nutzen kann. Das erfordert weniger ein Verständnis des „Innenlebens“ der relevanten Technologien, sondern vielmehr einen guten Überblick über aktuelle Innovationen, klare Vorstellungen über die Benutzung der informationstechnischen Werkzeuge sowie Grundkenntnisse des Technologie- und Innovationsmanagements.

Zusätzlich zum Wissen über das derzeitige und künftige Geschäft, die Technologiepotenziale und ihre Nutzung sowie Visionen neuer Geschäftslösungen benötigt ein Business Engineer die Fähigkeit, Menschen dazu zu motivieren, die neuen Lösungen zu verstehen, zu akzeptieren und das eigene Verhalten entsprechend zu verändern.

„Harte“ Wirtschafts- bzw. Management-Fachkenntnisse, Kreativität, Verständnis für IT-Innovationen und für „weiche“ Aspekte der Veränderung allein machen jedoch noch keinen Business Engineer aus: Ingenieurmässiges Handeln bedeutet, Probleme arbeitsteilig und transparent durch Anwendung geeigneter Methoden bzw. auf der Grundlage geeigneter Modelle zu lösen (Österle, 1995). Erst das Vorhandensein einer umfassenden methodischen Grundlage und die Fähigkeit zur Anwendung dieser Methoden machen aus Veränderungskünstlern, die intuitiv arbeiten, Veränderungsingenieure, die im Team komplexe Veränderungsprozesse konstruieren und umsetzen.

Damit sind die wesentlichen Qualifikationsbereiche des Business Engineers abgesteckt (Baumöl, Winter, 2003):

Betriebswirtschaftliche Fachkenntnisse (z.B. Vorstellungen über künftige Strukturen und Organisationsformen der Wirtschaft, Unternehmensführung, Organisationsentwicklung, Wirtschaftspolitik)

Anwendungsorientiertes Verständnis der Informations- und Kommunikationstechnologie (z.B. Kenntnis der aktuellen IT-Innovationslandschaft, Methoden des Technologie- und Innovationsmanagements) Verständnis für die politischen, kulturellen und emotionellen Aspekte von Veränderungsprojekten (z.B. Psychologie und Führung in Verän- 
derungsprojekten, Change Management, Schaffung bzw. Aufrechterhaltung der Rahmenbedingungen für Veränderungen in Unternehmen)

Führungsqualitäten (z.B. Fähigkeit zur Kommunikation von Visionen, zur Motivation und Führung von Mitarbeitern in der Veränderung und zu effektiver Teamarbeit)

Fähigkeit zur Anwendung von Transformationsmethoden (z.B. Ableitung von Geschäftslösungen mit Blick auf IT-Potenziale, Spezifikation von Geschäftsmodellen des Informationszeitalters, Optimierung von Geschäftsprozessen für bestimmte Geschäftsmodelle, Spezifikation von Informationssystemen für bestimmte Geschäftsprozesse)

Fähigkeit zur effektiven Sammlung, Nutzung und Weitergabe von Informationen und Wissen.

Business Engineers verbinden damit im Idealfall Fachkompetenz (d.h. Detailkenntnisse bzw. Detailverständnis der verschiedenen relevanten Theorien, Modelle und Aspekte des Wandels, Integrationswissen, das sich durch die umfassende, integrative Beschäftigung mit den unterschiedlichen Aspekten von Veränderungsprojekten ergibt und die Fähigkeit zur ingenieurmässigen Anwendung von Transformationsmethoden) mit Sozialkompetenz auf der Grundlage eines breiten Erfahrungswissens aus eigenen und fremden Erfahrungen in Veränderungsprojekten.

\section{Die Ausbildung zum Business Engineer}

In Anbetracht der Vielfältigkeit der im vorangehenden Abschnitt analysierten Qualifikationsbereiche kann sich eine Ausbildung zum Business Engineer nicht auf die Vermittlung isolierter Kenntnisse beschränken, sondern muss auf verschiedenen Säulen ruhen:

Vermittlung ausreichender Detailkenntnisse bzw. ausreichenden Detailverständnisses sowohl in „harten“ als auch „weichen“ Gegenstandsbereichen des Wandels. Fachliche Schwerpunkte in Betriebswirtschaftslehre, Technologie/Wirtschaftsinformatik und (Organisations-)Psychologie sind durch das Schaffen von Eindrücken / Erlebnissen sowie die Sensibilisierung für Politik- und Kulturaspekte zu ergänzen.

Schaffung von Gelegenheiten zur Bildung von Integrationswissen, z.B. im Rahmen eines die verschiedenen Gegenstandsbereiche übergreifenden Projekts oder einer intensiven Verknüpfung der verschiedenen Aspekte, sei es mit technischen Hilfsmitteln (Wissensplattform für Business Engineers) oder durch eine intensive Abstimmung der Lehreinheiten. 
Schaffung einer Vielzahl von Gelegenheiten zur Anwendung von Methoden, z.B. in Form von Fallstudien. Wenn möglich, sollten Fallstudien nicht einen Einzelaspekt der Thematik abdecken, sondern eine integrative Bearbeitung unter verschiedenen Perspektiven zulassen.

Gelegenheit zur Entwicklung bzw. Weiterentwicklung von Sozialkompetenz. Auch in der Ausbildung sind Teambildung und Teamarbeit möglichst realitätsnah, häufig und in wechselnden Teamkonstellationen einzusetzen. Das passiert in der „geschützten Testumgebung“ des Kurses, so dass z.B. auch die Thematik „Teamkonflikte“ direkt adressiert werden kann, ohne unmittelbare Auswirkungen, z.B. für die Karriere, befürchten zu müssen. Intensive Individual- und Gruppenerfahrungen können z.B. in Outdoor-Ausbildungseinheiten erworben werden.

Schaffung von Gelegenheiten zur Weitergabe von Erfahrungswissen, z.B. durch Gastvorträge von Veränderern, Analyse realer Veränderungsprojekte, gegenseitige Präsentation der eigenen Erfahrungen in selbst erlebten Veränderungsprojekten und gegenseitige Präsentation der unterschiedlichen Lösungsansätze für Übungen und Fälle.

Die hohen fachlichen und methodischen Ansprüche an Business Engineers schränken die in Frage kommenden Ausbildungsvarianten auf die tertiäre Ausbildung ein. Nur in einer Hochschule - und nicht an Fachschulen oder im Rahmen unternehmensinterner Programme - wird es im Normalfall möglich sein, die unterschiedlichsten Aspekte von Veränderungsprojekten integrativ, in ausreichender wissenschaftlicher Tiefe und ohne zu starke Einschränkung auf bestimmte, unternehmensspezifische Aspekte zu vermitteln. Darüber hinaus ist zu berücksichtigen, dass ein starker Praxisbezug hergestellt werden sollte und für die Vertiefung der vermittelten Inhalte eine intensive Interaktion zwischen den Vertretern aus der Praxis und den Auszubildenden stattfinden muss.

Das (Erst-)Hochschulstudium eignet sich deshalb nur sehr beschränkt für die Ausbildung von Business Engineers. Im Normalfall kann nicht auf ausreichenden Erfahrungen der Studierenden aufgebaut werden. Darüber hinaus lässt die traditionelle Form der öffentlichen Finanzierung des Hochschulstudiums Interaktionen mit Studierenden nicht in der als notwendig erachteten Intensität zu, da in der Regel die Mittel für einen hohen Betreuungsgrad nicht zur Verfügung stehen.

Studierende eines Vollzeit-Nachdiplomstudiums hingegen bringen bei entsprechender Zulassungspolitik die notwendigen Erfahrungen mit. Das Vollzeitstudium zwingt allerdings dazu, die Studierenden aus ihrer beruflichen Aufgabe „herauszureissen“ und verschenkt somit die Möglichkeit, durch Abwechslung von Ausbildungsblöcken und beruflicher Tätigkeit Reflexionspotenziale zu schaffen und aufeinander aufbauende Anwendungserfahrungen zu sammeln. 
Zwar dauert ein berufsbegleitendes Nachdiplomstudium länger als ein Vollzeitstudium, erscheint aber als einzige Form in der Lage zu sein, Vorerfahrungen der Teilnehmer mit Gelegenheiten zu Integrationsprojekten und Reflexionsmöglichkeiten im ausgeübten Beruf zu kombinieren.

\section{Erfahrungen aus der Durchführung eines berufsbegleitenden Nachdiplomstudiums in Business Engineering}

An der Universität St. Gallen besteht seit 1998 ein berufsbegleitendes Nachdiplomstudium in Business Engineering, das in zwanzig Präsenzwochen (zuzüglich Anfertigung der Diplomarbeit) zum akademischen Abschluss „Executive MBA in Business Engineering“ führt. Die theoretischen Grundlagen des Programms und das sich daraus ergebende Curriculum werden z.B. bei Baumöl und Winter (2003) ausführlich beschrieben. Eingangsvoraussetzungen sind neben einem erfolgreich abgeschlossenen Hochschulstudium einschlägige Berufserfahrungen und die erfolgreiche Absolvierung einer Eingangsprüfung.

Andere Ausbildungsangebote, welche die Veränderungsproblematik adressieren, finden sich in der Regel ausserhalb von Hochschulen, immer aber mit wesentlich kürzerer Dauer und in der Regel ohne Berücksichtigung der speziellen Lernsituation von Teilnehmern mit unterschiedlichem Ausbildungshintergrund. Darüber hinaus integriert kein anderes Angebot „weiche“ Faktoren und Führungsaspekte annähernd in dem Umfang, den die Diskussion des Berufsbilds des Business Engineers weiter oben erfordert.

Für die 45 Studienplätze pro Jahr (Studienbeginn ist jeweils im Februar) liegen jeweils zwischen 60 und 110 qualifizierte Bewerbungen vor. Aufgrund der grossen Nachfrage wurden im Jahr 2001 zwei Durchführungen angeboten. Im Verlauf der letzten Jahre verschob sich die Finanzierung des Programms von Mischformen (z.B. Übernahme von 50\% der Lehrgangsgebühr, von $50 \%$ des Zeitbedarfs und $50 \%$ der Reise- bzw. Aufenthaltskosten durch den Arbeitgeber) mehr und mehr zu einer vollständigen Finanzierung durch die Studierenden. Zwischen $75 \%$ and $90 \%$ der Studierenden kommen aus der Schweiz, der Rest vorwiegend aus Deutschland.

In Anbetracht der Ausrichtung des Programms auf das Berufsbild des Business Engineers überrascht es nicht, dass fast 50\% der Studierenden einen betriebswirtschaftlichen Erstabschluss haben. Hinsichtlich der Branchenherkunft arbeiten mit knapp 40\% die meisten Studierenden im Fi- 
nanzdienstleistungsbereich. Detaillierte Angaben zur Vorbildung, Tätigkeit und zur Karrierewirkung des Programms finden sich bei Winter (2002).

Auf Grundlage der Selbstevaluation der ersten Durchführungen durch die Studienleitung und der sehr ausführlich erhobenen Evaluationen der Studierenden wurden verschiedene inhaltliche Anpassungen vorgenommen. So wurde z.B. anstelle eines Einstiegs in den Lehrgang auf der Basis rein fachlicher Inhalte eine Kick-off-Woche konzipiert, die sowohl fachliche als auch teamentwicklungsorientierte Elemente verbindet. Auf diese Weise werden erste Teamprozesse in der neu zusammengekommenen Gruppe angestossen, die ohne eine explizite Adressierung nicht stattfinden würden. Gleichzeitig werden die grundlegenden Konzepte des Business Engineering vermittelt. Eine weitere, aus der Perspektive der Konsistenz der Lehrgangsinhalte wichtige Anpassung, ist die Umstrukturierung der letzten Woche als Fachthema-übergreifende Zusammenführung wesentlicher Aspekte der gesamten Ausbildung. Die Einführung von Wahlmöglichkeiten wurde zugunsten einer deutlicheren Strukturierung des Programms in „allgemeine“ MBA-Inhalte einerseits und spezielle Business Engineering-Inhalte andererseits wieder aufgegeben.

Die Weiterbildung im Erwachsenenbereich und hier insbesondere die akademische Weiterbildung mit dem Ziel, ein akademisches Diplom zu erwerben, hat sich immer weiter in Richtung der Philosophie des „lebenslangen Lernens" bewegt. Deshalb werden entsprechende Ausbildungen immer häufiger durch sogenannte „Refresher“ oder andere regelmässige Seminare und Weiterbildungsveranstaltungen fortgeführt. Der Abschluss eines solchen Nachdiplomstudiums ist immer zugleich auch der Anfang einer aktiven Wissensgestaltung und Wissensentwicklung. Nicht zu vernachlässigen ist auch die Fortführung und Pflege der während des Studiums begonnenen Bildung eines beständig wachsenden Beziehungsnetzwerkes, das wiederum den Wissensaustausch fördert.

Besonderer Wert wurde auf die Entwicklung eines studien- und berufsbegleitenden Intranets, das „Business Engineering Center“, gelegt. Diese Plattform dient in den Zeiten zwischen den Lehrgangsmodulen und für die Zeit nach Abschluss des Programms als Kommunikations- und Arbeitsplattform und unterstützt damit nicht nur räumlich verteilte Teamarbeit, sondern auch die Entwicklung einer Business Engineering-Community. Die Entwicklung eines solchen Netzwerks ist neben dem intensiven Erfahrungsaustauschs eine der grundlegenden Zielsetzungen eines Studiums zum Veränderungsspezialisten.

In Zukunft werden Community-Plattformen,. Alumni-Veranstaltungen und ähnliche Konzepte in der tertiären Bildung immer mehr an Bedeutung gewinnen. Im Zuge der Globalisierung berufsbegleitender Studiengänge liegt auch hier der Schlüssel zum Erfolg in der effektiven Nutzung der zur 
Verfügung stehenden Technologien und damit der Transformation des Geschäftsmodells im Sinne des Business Engineering.

\section{Literatur}

[Baumöl, Winter, 2003]

Baumöl, U.; Winter, R.: Qualifikation für die Veränderung, in: Österle, H.; Winter, R. (Hrsg.): Business Engineering - Auf dem Weg zum Unternehmen des Informationszeitalters, 2. Aufl., Berlin etc.: Springer 2003, S.45-61.

[Davenport, 1993]

Davenport, P. H., Process Innovation: Reengineering Work through Information Technology, Boston: Harvard Business School Press, 1993.

[Evans, Wurster 1999]

Evans, P., Wurster, T.S., Blown to Bits - How the new Economics of Information transforms Strategy, Boston: Harvard Business School Press, 1999.

[Kelly 1998]

Kelly, K., New Rules for the new Economy - 10 radical Strategies for a connected World, New York: Viking Penguin, 1998.

[Martin 1995]

Martin, J., The Enterprise Engineer, Computerworld, 18.9.1995.

[Österle 1995]

Österle, H., Business Engineering, 2. Aufl., Springer, Berlin et al., 1995.

[Österle, Fleisch, Alt 2001]

Österle, H., Fleisch, E., Alt, R., Business Networking in der Praxis, Springer, Berlin et. al, 2001.

[Österle, Winter 2003]

Österle, H., Winter, R., Business Engineering - Auf dem Weg zum Unternehmen des Informationszeitalters, 2. Aufl., Springer, Berlin et al., 2003.

[Winter 2002]

Winter, R., An Executive MBA Program in Business Engineering: A Curriculum Focusing on Change, Journal of IT Education, Vol. 1 (2002), No. 4, pp. 279-288. 